\title{
Network pharmacology to dissect the mechanisms of Yinlai Decoction for pneumonia
}

Jingnan $\mathrm{Xu}^{2 \dagger}$, Chen Bai ${ }^{1 \dagger}$, Ling Huang ${ }^{1 \dagger}$, Tiegang Liu ${ }^{1 \dagger}$, Yuxiang Wan², Zian Zheng ${ }^{3}$, Xueyan Ma', Fei Gao', $\mathrm{He} \mathrm{Yu}^{1}$ and Xiaohong $\mathrm{Gu}^{1 *}$

\begin{abstract}
Background: Pneumonia is a common respiratory disorder, which brings an enormous financial burden to the medical system. However, the current treatment options for pneumonia are limited because of drug resistance and side effects. Our previous study preliminarily confirmed that Yinlai Decoction (YD), a common prescription for pneumonia in clinical practice, can regulate the expression of inflammatory factors, but the mechanisms are unknown yet.

Methods: In our work, a method named network pharmacology was applied, which investigated the underlying mechanisms of herbs based on a variety of databases. We obtained bioactive ingredients of YD on TCMSP database and collected potential targets of these ingredients by target fishing. Then the pneumonia-related targets database was built by TTD, Drugbank, HPO, OMIM, and CTD. Based on the matching targets between YD and pneumonia, the PPI network was built by STRING to analyze the interactions among these targets and then input into Cytoscape for further topological analysis. DAVID and KEGG were utilized for GO and pathway enrichment analysis. Then rat model based on LPS stimulated pneumonia was used to verify the possible mechanism of YD in treating pneumonia.

Results: Sixty-eight active ingredients, 103 potential targets and 8 related pathways, which likely exert a number of effects, were identified. Three networks were constructed using Cytoscape, which were herb-component-network, YD-pneumonia target network, and herb-component-YD target-pneumonia network. YD was verified to treat LPSinduced pneumonia by regulating the inflammatory factor IL-6, which was a predicted target.

Conclusion: Network analysis indicated that YD could alleviate the symptoms and signs of pneumonia through regulating host immune inflammatory response, angiogenesis and vascular permeability, the barrier function of the airway epithelial cells, hormone releasing and cell growth, proliferation, and apoptosis.
\end{abstract}

\footnotetext{
* Correspondence: guxiaohong1962@163.com

†Jingnan Xu, Chen Bai, Ling Huang and Tiegang Liu contributed equally to this work.

'School of Traditional Chinese Medicine, Beijing University of Chinese Medicine, Beijing 100029, People's Republic of China

Full list of author information is available at the end of the article
}

(c) The Author(s). 2020 Open Access This article is licensed under a Creative Commons Attribution 4.0 International License, which permits use, sharing, adaptation, distribution and reproduction in any medium or format, as long as you give appropriate credit to the original author(s) and the source, provide a link to the Creative Commons licence, and indicate if changes were made. The images or other third party material in this article are included in the article's Creative Commons licence, unless indicated otherwise in a credit line to the material. If material is not included in the article's Creative Commons licence and your intended use is not permitted by statutory regulation or exceeds the permitted use, you will need to obtain permission directly from the copyright holder. To view a copy of this licence, visit http://creativecommons.org/licenses/by/4.0/ The Creative Commons Public Domain Dedication waiver (http://creativecommons.org/publicdomain/zero/1.0/) applies to the data made available in this article, unless otherwise stated in a credit line to the data. 


\section{Background}

Globally, pneumonia is one of the leading causes of morbidity and mortality and accounted for 3 million deaths worldwide in 2016 [1], and imparts an enormous financial burden to the medical system [2]. The coronavirus disease 2019 (COVID-19) pneumonia has been declared a pandemic by the World Health Organization (WHO) on March 11, 2020 [3]. It has triggered enormous human casualties and serious economic loss and attracted the world's enormous attention to pneumonia. Pneumonia is a syndrome with common clinical symptoms such as fever, cough, dyspnea, and fixed moist rale in the lungs, primarily caused by host immunoreaction to respiratory pathogens including bacteria, virus, mycoplasma, and chlamydia, etc. Currently, the main treatments for pneumonia include antibacterial, antivirus, immune inhibition, analgesics, antipyretics, and antihistamine. However, due to drug resistance and the limited efficacy of specific symptoms, their clinical application is limited [4].

Traditional Chinese medicine (TCM) holds a holistic and integrative perspective on health maintenance. Therapeutic effect of Chinese herbal represents the outcome achieved from the combined effects of multitargets and multi-pathways, which help to reach a state of individual internal physiological harmony. Yinlai Decoction (YD) comprises seven herbs: Lonicera japonica Thunb (L. japonica Thunb, JYH), Raphanus sativus $L$ ( $R$. sativus L, LFZ), Forsythia suspensa (Thunb.) Vahl (F. suspensa, LQ), Scutellaria baicalensis Georgi (S. baicalensis Georgi, HQ), Peucedanum praeruptorum Dunn (P. praeruptorum Dunn, QH), Houttuynia cordata Thunb (H. cordata Thunb, YXC), Trichosanthes rosthornii Harms (F. rosthornii Harms, GL), and is commonly used for respiratory tract infection in clinical practice, such as influenza and pneumonia. Our previous study preliminarily confirms that YD can alleviate the symptoms of influenza virus infection and regulate the level of inflammatory factors such as interleukin (IL)-10 and tumor necrosis factor (TNF)- $\alpha$ [5]. However, the effective components and the mechanisms of YD in the treatment of pneumonia were still unclear and further exploration is necessary.

Network pharmacology is characterized by discovering TCM from a systematic perspective and at the molecular level. A growing evidence shows that the network pharmacology strategy can be a powerful approach to modern research on TCM [6, 7]. Based on the interaction network analysis of "disease-target-drug", the intervention and influence of drugs on disease are systematically and comprehensively observed [8]. Comparing with the classic trial and error method, network pharmacology can provide a system-level of understanding the interaction mechanism between drugs and complex diseases in a high-throughput manner and within less time. Different from the current"one target, one drug" paradigm, the mechanisms of "multi-targets and multi-components" on the disease can be revealed based on this approach [5]. In brief, network pharmacology is an attractive modality that assists in drug development [9].

In this work, active ingredients prediction, target fishing, and network construction were carried out to evaluate active ingredients and targets of YD, so as to reveal the mechanisms of YD for pneumonia and provide a novel method for clinical practice.

\section{Methods}

The basic process of network pharmacology includes: identification of ingredients in the compound, acquisition of disease-related targets, construction of compound-ingredient-target-disease network, bioinformatics annotation and experimental verification. The visible procedure of analysis for the mechanism of YD for pneumonia based on network pharmacology was as follows (as displayed in Fig. 1).

\section{Data preparation}

Totally, 625 chemical ingredients of 7 herbs in YD were manually extracted from Traditional Chinese Medicine Systems Pharmacology Database (TCMSP, http://lsp. nwu.edu.cn/tcmsp.php, Version 2.3). TCMSP have collected all the herbs registered in Chinese pharmacopoeia (2010) based on critically examined pharmacology and clinical knowledge, and is a pharmacological platform of TCM which covered a variety of pharmacokinetic parameters, targets, and related diseases [10].

\section{Bioactive ingredients prediction}

ADME (absorption, distribution, metabolism, and excretion) evaluation system can save the time and cost of the experiments by screening the active ingredients employing the above stated databases. Thus, three pharmacokinetic parameters including oral bioavailability (OB), Caco-2 cell permeability (Caco-2) and drug-likeness (DL) were selected to identify the active ingredients of YD.

$\mathrm{OB}$ refers to oral bioavailability, which is defined as the rate of active ingredient being absorbed from a drug product and becoming available at the site of action [11]. Caco-2 permeability is widely used to predict the intestinal ability to absorb components [12]. DL is a qualitative concept used in drug design and applied to assess the balance among molecular properties, which can explain how "druglike" a compound is [13]. In this study, $\mathrm{OB} \geq 30 \%$, Caco- $2>-0.4$ and $\mathrm{DL} \geq 0.18$ were the screening criteria for our ADME evaluation system. The components qualified for these criteria were regarded as the candidate ingredients for follow-up analysis. 


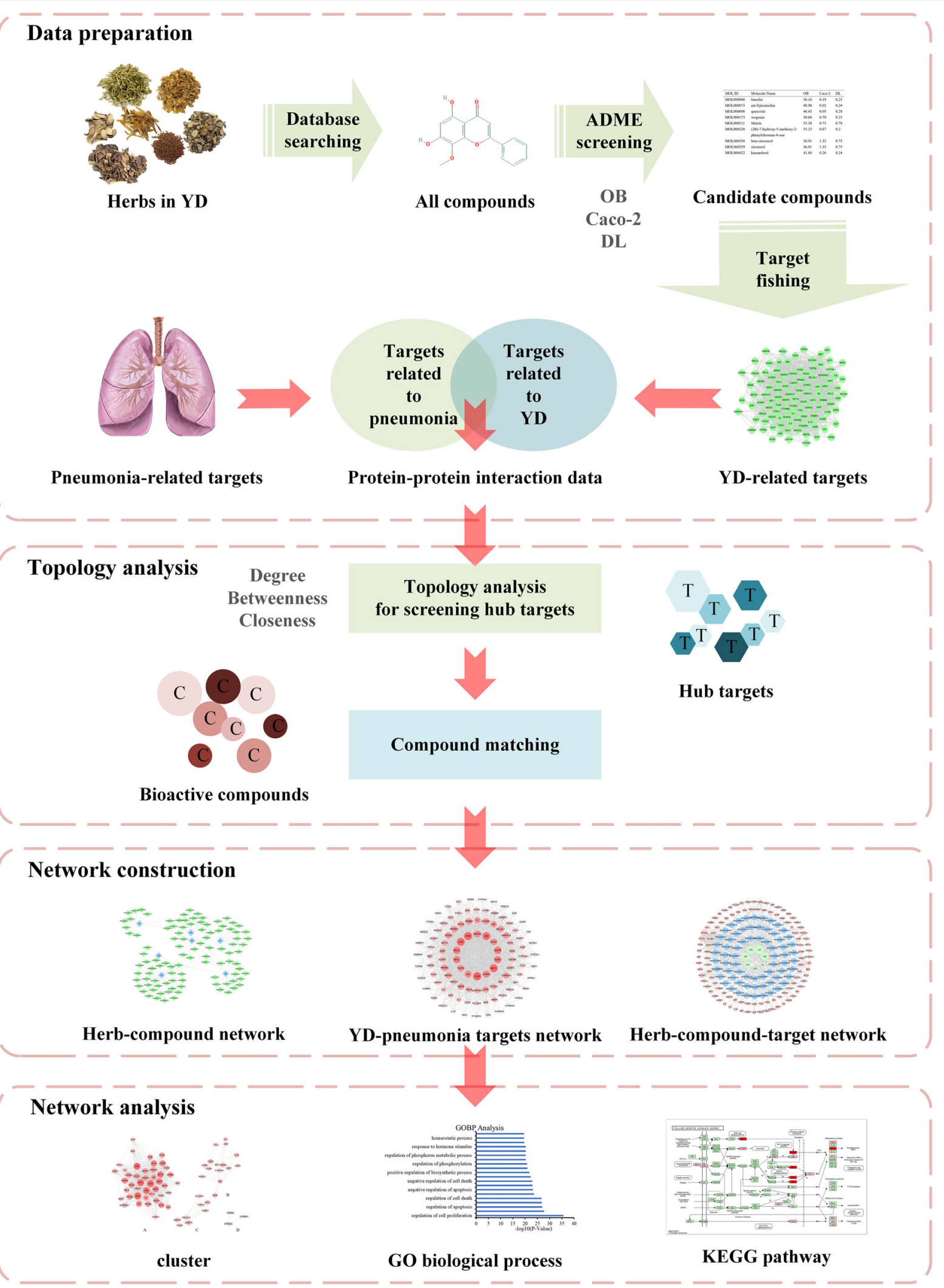

Fig. 1 Flow chart of pharmacological analysis of YD for pneumonia treatment 
Thirty-six molecules were obtained from $S$. baicalensis Georgi, 23 from L. japonica Thunb, 21 from F. suspensa, 18 from P. praeruptorum Dunn, 11 from F. rosthornii Harms, 6 from $H$. cordata Thunb, and 3 from $R$. sativus $L$. Many overlapping molecules among the seven herbs were removed.

\section{Target fishing}

We input all the bioactive components into TCMSP and obtained the targets of each component. Several components had no relevant targets, such as (MOL000358) betasitosterol, (MOL000359) sitosterol, (MOL000449) Stigmasterol, (MOL002926) dihydrooroxylin A, (MOL003365) Lactucasterol, (MOL003281) 20(S)-dammar-24-ene-3ß,20-diol3-acetate, (MOL003975) icosa-11,14,17-trienoic acid methyl ester and (MOL013101) rutarin_qt, which were drawn in ChemBioDraw version 16, saved as "mol2" file and imported into ChemMapper (http://lilab.ecust.edu.cn/ chemmapper/, entering at Jan 2018). It is a website that discovers drug through computer methods in view of the notion that ingredients with similar structures may have similar targeting properties [14]. The Universal Protein Resource Knowledge base (UniProt Knowledge base, http:// www.uniprot.org/, entering at Jan 2018) is applied to unify the standard nomenclature, which is a collection of information of functional proteins with accurate, consistent and rich annotation [15]. Filtered by "swiss-prot reviewed" and "Homo sapiens", the official symbol of these proteins was acquired. By far, we acquired the bioactive components and potential targets of YD.

\section{Pneumonia targets}

Genes related to pneumonia were acquired from the following existing databases. The former two databases were used to collect relevant targets for drugs currently on the market or under research to treat pneumonia. The latter three databases were used to collect as comprehensive a collection of pneumonia-related targets as possible from authoritative databases. (1) Therapeutic Target Database (TTD, https://db.idrblab.org/ttd/, version Sep 15th, 2017) - a database for collecting information on 2544 approved drugs, 8103 drugs in clinical trials and 18,923 drugs under investigation [16]. (2) Drugbank (https://www.drugbank.ca/, version 5.1.1) - a database for collecting information on 4100 approved or experimental drug products [17]. (3) Human Phenotype Ontology (HPO, http://human-phenotype-ontology. github.io/, entering at Jan 2018) - a database for collecting information on genetic phenotypes in human disease [18]. (4) Online Mendelian Inheritance in Man (OMIM, http://omim.org/, entering at Jan 2018) - a database for collecting comprehensive compendium of human genes [19]. (5) The Comparative Toxicogenomics Database (CTD, http://ctdbase.org/, entering at Jan 2018) - a database for collecting phenotype human disease [20]. The latter three databases are all authoritative databases which collect pneumonia-related phenotype. We retrieved these databases with a keyword "pneumonia" and obtained 51,136 genes in total.

\section{Protein-protein interaction data}

PPI data on 296 common targets between YD and pneumonia were extracted from STRING (http://string-db. org/, v. 10) with parameters filtered by "Homo sapiens" (confidence score $>0.4$ ), which is a database covering no less than 2000 organisms and constructing interaction network between proteins by novel algorithms [21]. Subsequently, the data description of the correlation between every two targets, defined as the score, was acquired.

\section{Topological analysis of YD-pneumonia target network}

Data from STRING were input into Cytoscape version 3.6.0 for topological analysis. Cytoscape is designed to visualize the network of biological pathways and intermolecular interaction networks. Moreover, topological parameters can be measured by Network Analyzer, a plug-in of Cytoscape, for complicated network analysis [22]. Finally, we obtained 103 hub targets with 3 key topological parameters as a degree, closeness and betweenness centralities larger than the median, and matched them with the candidate components. Thus, 68 bioactive ingredients were correlated with 103 hub targets from YD and pneumonia. The PPI analysis was performed as described above.

\section{Network construction}

First, we built an interaction network of the related herbs and bioactive components in YD. Then, the interaction between potential common YD-related targets and pneumonia-related targets were obtained based on PPI data and topological analysis. Finally, a holistic network of herbs, components, and targets of YD-pneumonia was constructed.

\section{Network analysis \\ Cluster}

In protein interaction networks, topological modules or clusters represent dense regions of highly correlated molecular components in pure network property. The clusters were extracted by calculating the corresponding networks with Molecular Complex Detection. Finally, we obtained 4 clusters of highly interconnected targets.

\section{Gene ontology and KEGG pathway enrichment}

Clusters were input into The Database for Annotation, Visualization and Integrated Discovery (DAVID, https:// david-d.ncifcrf.gov/, version 6.7) [23] for Gene ontology 
(GO) and Kyoto Encyclopedia of Genes and Genomes (KEGG, https://www.kegg.jp/, entering at Jan 2018) for pathway enrichment analysis. Several pneumonia-related biological processes and pathways were returned. The biological processes and pathways with Bonferroni $<0.05$ were considered to be significant. To further uncover the therapeutic mechanism of YD, we identified 8 pathways associated with pneumonia mechanism.

\section{Experiment validation \\ Animals and models}

Eighteen Clean grade Sprague-Dawley (SD) rats, male, 4weeks-old, weighing $110 \pm 10 \mathrm{~g}$, were purchased from Sibeifu (Beijing) Biotechnology Co., Ltd., license No.: SCXK (Jing) 2016-0002 (animal certificate No.: $1103241800000595)$. They were raised in the animal room of the Beijing University of traditional Chinese medicine with free access to food and water, natural light. The rats were randomly divided into three groups: normal control group (NC), pneumonia group (P), pneumonia treatment group (PT), 6 rats in each group. Rats were adaptively bred for 3 days before starting the experiment. From the first day of formal experiment, all groups were given ordinary feed. P group was sprayed with $5 \mathrm{ml}$ LPS solution $(0.5 \mathrm{mg} / \mathrm{ml})$ one time a day for $30 \mathrm{~min}$. NC group and PT group were given equal dose of pure water atomization. The rats in PT group were administrated YD ( $2 \mathrm{ml} / 100 \mathrm{~g}$ ) by means of intragastric administration contrasting with normal saline $(2 \mathrm{ml} / 100 \mathrm{~g})$ given to rats in NC group and $\mathrm{P}$ group. After 3 days' model building, all the rats were fast but free for water. On the fourth morning, the rats were anesthetized by intraperitoneal injection of $10 \%$ chloral hydrate $(0.2 \mathrm{ml} / 100 \mathrm{~g})$. The left lung was removed and placed in a $4 \%$ formaldehyde fixative, and stored at $4{ }^{\circ} \mathrm{C}$, while the right lung placed in a frozen pipe and frozen by liquid nitrogen, then stored at $-80^{\circ} \mathrm{C}$.

\section{Chemical drug and agents}

Lipopolysaccharides (LPS from Eschericia coli 055: B5, $100 \mathrm{mg}, 046 \mathrm{~m} 4045 \mathrm{v}$, sigma company, USA). ELISA Kit for Interleukin 6 (IL6) (SEA079Ra, 96 t, Cloud-Clone Corp., USA).

\section{Tissue detection}

The transverse sections of the left lung tissues were prepared according to the routine procedure for tissue wax block-slice-hematoxylin-eosin (HE) staining. The right lung tissue homogenate was prepared and detected by ELISA method according to instruction.

\section{Results}

Herb-component network of YD

Generally, herbs are administered through oral route and eventually enter the blood circulation where they are maintained at a certain concentration gradient that has a pharmacological effect on the patient [24]. After administration, the concentration of the bioactive ingredients at the target site is controlled by the pharmacokinetic process, which ultimately affects the therapeutic response [25]. Bearing this in mind, we opted for three classical ADME properties which were OB, Caco-2 and DL to filter the bioactive components of YD. Sixty-eight components related to pneumonia and having good ADME properties were obtained (as shown in Supplementary Information Table S1) from database search. The top-listed compounds were ketones/flavonoids (52.94\%), followed by coumarins (14.71\%), esters (13.24\%), alcohols (10.29\%), phenols (4.41\%), alkaloids (1.47\%), acids $(1.47 \%)$, and olefins $(1.47 \%)$ (as displayed in the Fig. 2). Nine components were involved in more than one herb, including luteolin, quercetin, wogonin, beta-sitosterol, sitosterol, kaempferol, mandenol, stigmasterol and spinasterol, 8 of which belong to ketones or flavonoids. The connection was established between components and herbs in YD (as displayed in Fig. 3).

\section{YD-pneumonia target network}

The topological analysis was performed to uncover the properties of the proteins and to explore key therapeutic targets of YD. There are different features to represent the position of the nodes in an interaction network, such as average shortest path length, betweenness centrality, closeness centrality, clustering coefficient, degree and topological coefficient. Among them, degree, closeness, and betweenness centrality are used to evaluate the centrality of nodes in the network [26]. It is an important characteristic of large-scale networks because degree is the number of connections to a node and closeness is used to measure the degree of centralization. The node with higher closeness centrality goes through a shorter way to the center. Betweenness centrality shows how frequently a node flows on all shortest paths. As the betweenness centrality is higher, the more important a node is [27-29]. We defined nodes with the degree, closeness, and betweenness centrality all above the median as hub targets, which occupies the central position of the network. We searched "pneumonia" as a keyword in Therapeutic Target Database (TTD), Human Phenotype Ontology (HPO), Online Mendelian Inheritance in Man (OMIM), The Comparative Toxicogenomics Database (CTD), and finally obtained 51,136 related targets, and 296 of them overlapping to YD related targets. After topological eigenvalue calculation, we selected 103 hub targets and 1897 edges (as shown in Supplementary Information Table S2). Finally, we constructed a targettarget network based on the overlapping data between YD-related targets and pneumonia-related targets, 


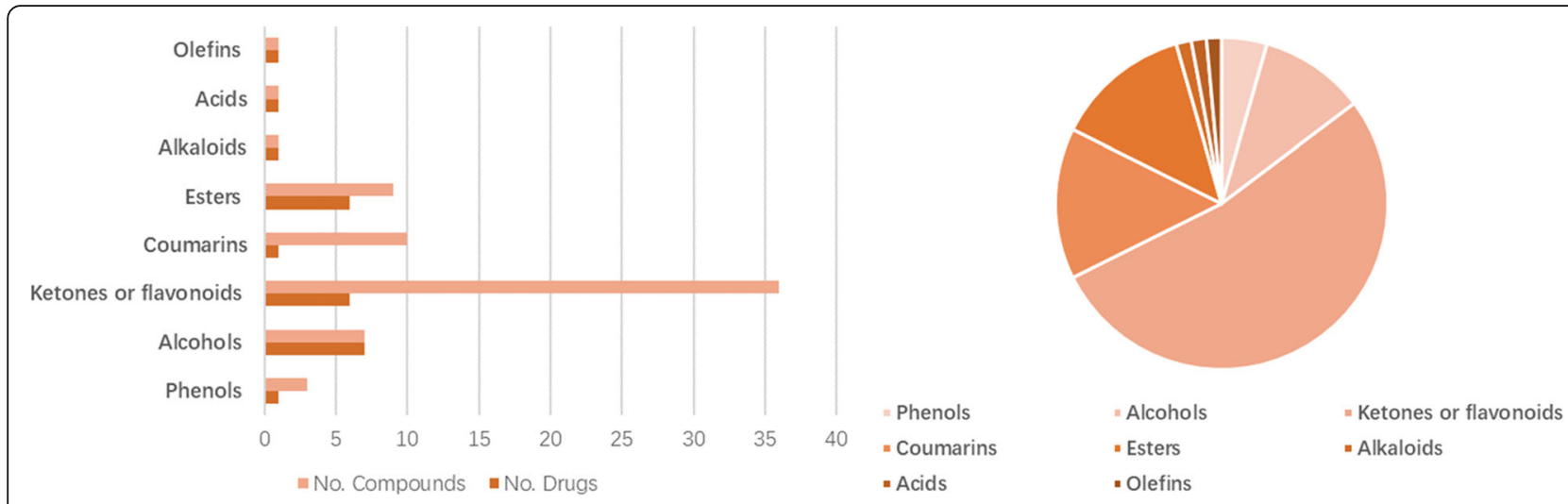

Fig. 2 Number and percentage of different active compounds of YD

indicating potential pharmacological functions in YD for treating pneumonia (as displayed in Fig. 4).

\section{Herb-component-target of YD-pneumonia network}

We constructed an "herb-component-target of YDpneumonia" network. It showed that an herb could interact with multiple components, and a component could also target several targets related to pneumonia (as shown in Supplementary Information Table S3). In the sequence of importance, the herbs in YD were as follows: LQ ( $F$. suspensa, degree $=67)$, YXC $(H$. cordata Thunb, degree $=$ 59), QH (P. praeruptorum Dunn, degree = 53), JYH (L. japonica Thunb, degree = 47), HQ (S. baicalensis Georgi, degree $=45)$, GL (F. rosthornii Harms, degree $=8$ ), and LFZ ( $R$. sativus $L$, degree $=4$ ). Arranged by the importance, the targets were as follows: prostaglandin $\mathrm{G} / \mathrm{H}$ synthase 1

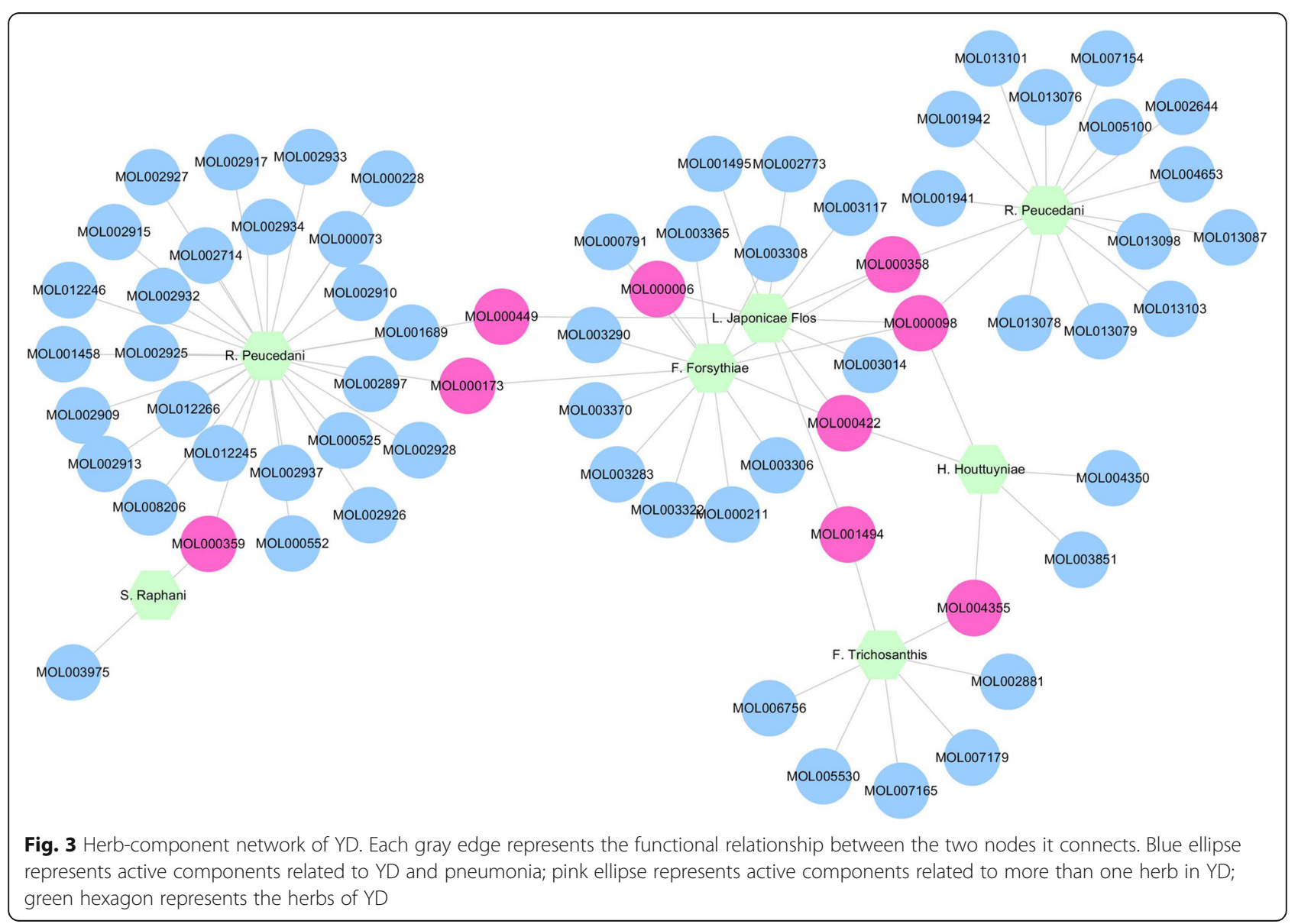




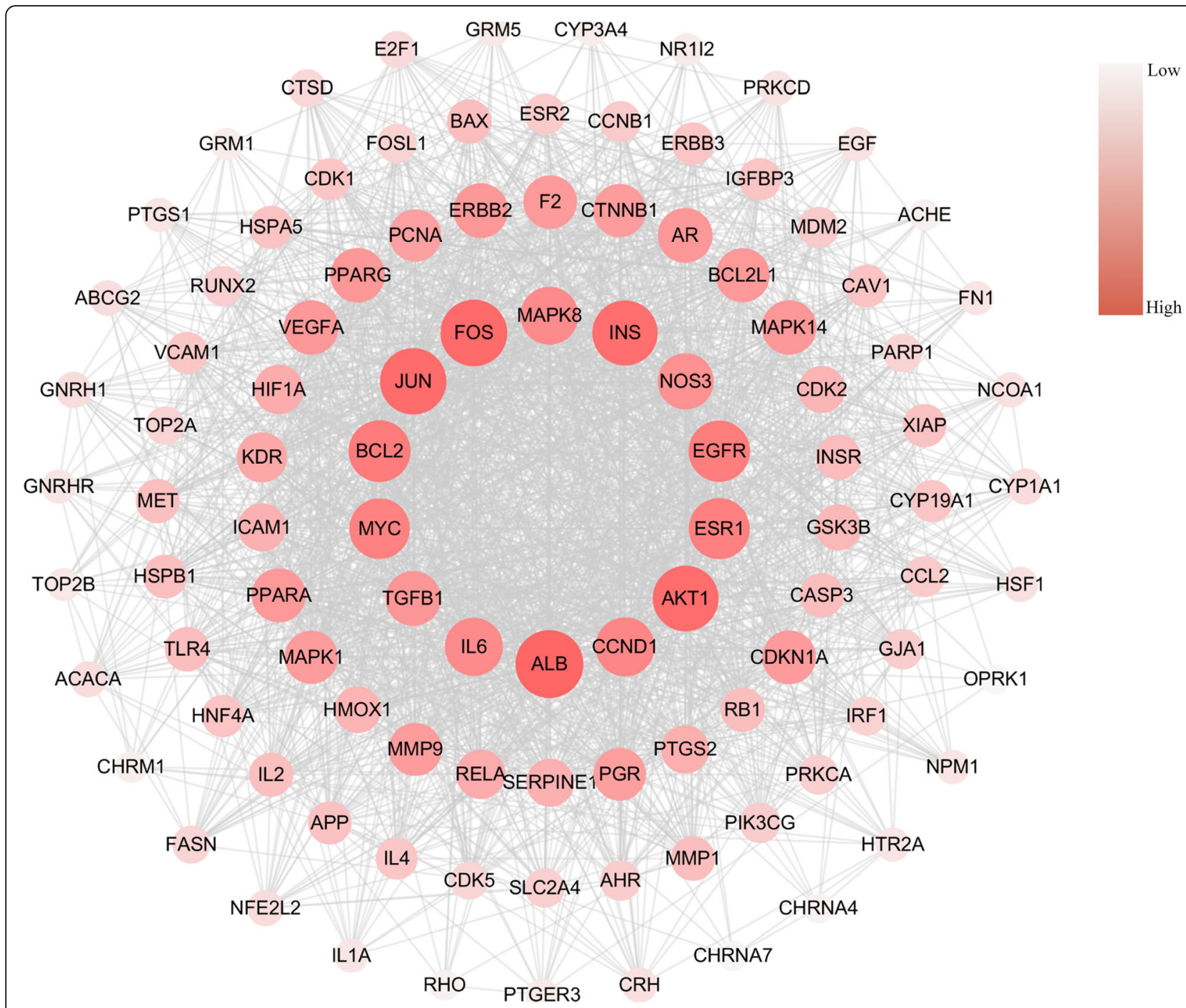

Fig. 4 YD-pneumonia target network. Each gray edge represents the functional relationship between the two nodes it connects. Each red ellipse represents targets related to both YD and pneumonia. The size and color depth of nodes are positively correlated with their degrees, respectively

(PTGS1, degree $=32$ ), prostaglandin $\mathrm{G} / \mathrm{H}$ synthase 2 (PTGS2, degree $=46)$, nuclear receptor coactivator 1 $(\mathrm{NCOA} 1$, degree $=21)$, androgen receptor $(\mathrm{AR}$, degree $=$ 19), phosphatidylinositol 4,5-bisphosphate 3-kinase catalytic subunit gamma (PIK3CG, degree = 16), estrogen receptor $2(E S R 2$, degree $=10)$ and so forth. It demonstrated that the ingredients of YD might play a "multi-targets and multi-components" therapeutic effect in the intervention of pneumonia-related targets (as displayed in Fig. 5).

\section{YD-pneumonia network analysis}

We analyzed the network with a cluster search algorithm, which considers highly connected dense regions as a network (Molecular Complex Detection), and four clusters were returned (as displayed in Fig. 6). Then, clusters were input into DAVID and KEGG for GO and pathway enrichment analysis, and only clusters A and B were involved in biological processes and pathways associated with pneumonia. According to the pathogenesis of pneumonia, these biological processes and pathways could be divided into three modules: (1) immunity and inflammation; (2) angiogenesis and epithelial cell proliferation; (3) cell growth, proliferation and apoptosis (as displayed in the Figs. 7 and 8).

\section{Immunity and inflammation}

Several biological processes associated with immunity and inflammation were returned and showed as follows: (GO: 0002520) immune system development, (GO:0002684) positive regulation of immune system process, (GO:0002697) regulation of immune effector process, (GO:0006954) inflammatory response, (GO:0006954) inflammatory response, (GO:0001817) regulation of cytokine production, (GO: 0042110) T cell activation, (GO:0002521) leukocyte differentiation, (GO:0001776) leukocyte homeostasis, (GO:0032496) 


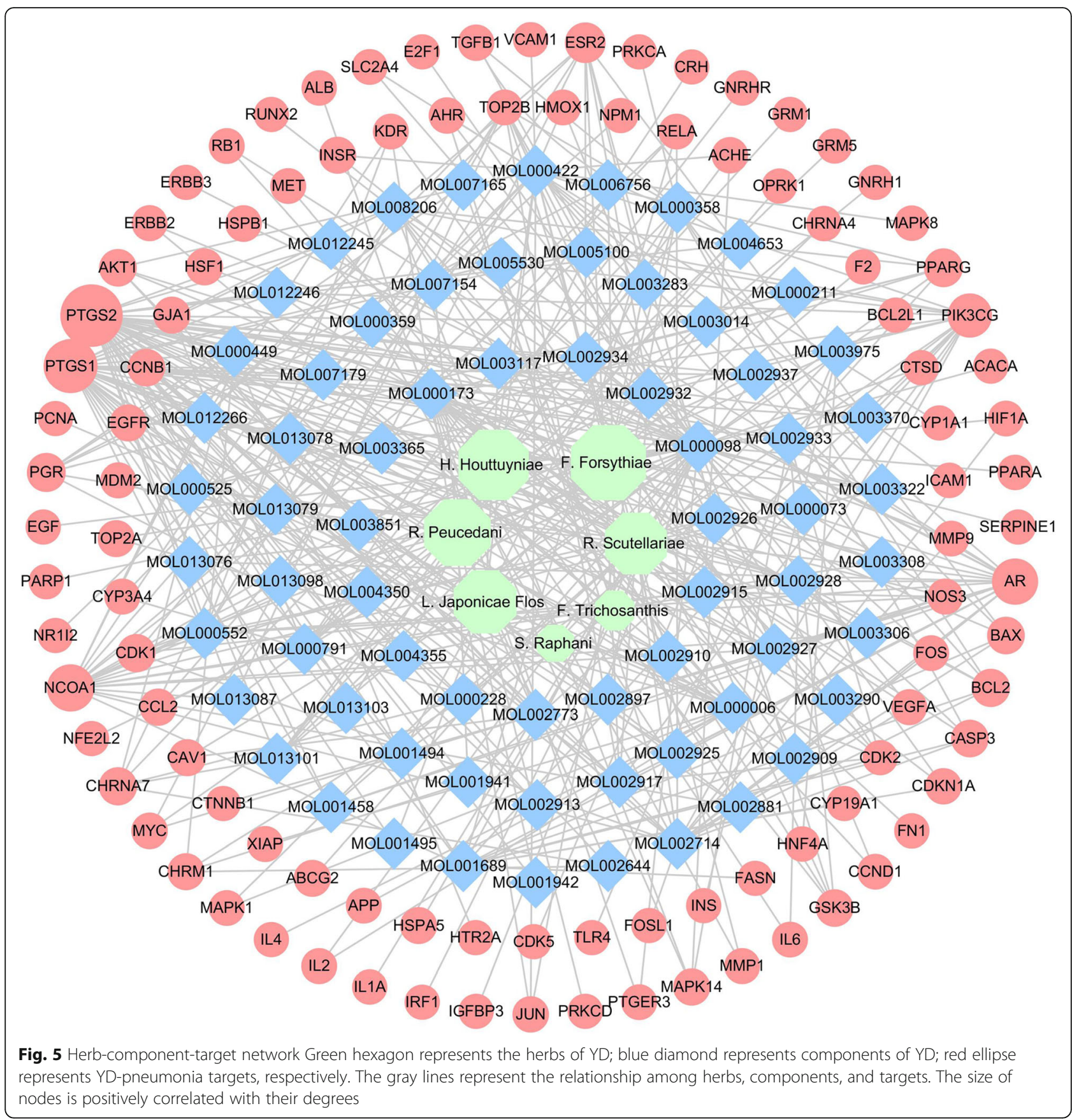

response to lipopolysaccharide and so forth (Bonferroni< 0.05). Additionally, three high-degree pathways were captured to be closely related to immunity and inflammation such as (hsa04620) Toll-like receptor signaling pathway (degree $=8$ ), (hsa04621) NOD-like receptor signaling pathway (degree $=6$ ), and (hsa04660) $\mathrm{T}$ cell receptor signaling pathway $($ degree $=7)$.

\section{Angiogenesis and epithelial cell proliferation}

In our work, some biological processes related with angiogenesis and epithelial cell proliferation were returned such as (GO:0001568) blood vessel development, (GO:0001944) vasculature development, (GO: 0048514) blood vessel morphogenesis, (GO:0050678) regulation of epithelial cell proliferation, (GO:0050679) positive regulation of epithelial cell proliferation, (GO: 0001525) angiogenesis and (GO:0045765) regulation of angiogenesis (Bonferroni< 0.05 ). Additionally, three high-degree pathways, (hsa04012) ErbB signaling pathway (degree $=9)$, (hsa04370) VEGF signaling pathway (degree $=8$ ), and (hsa05120) epithelial cell signaling in Helicobacter pylori infection (degree $=6$ ) were detected. 


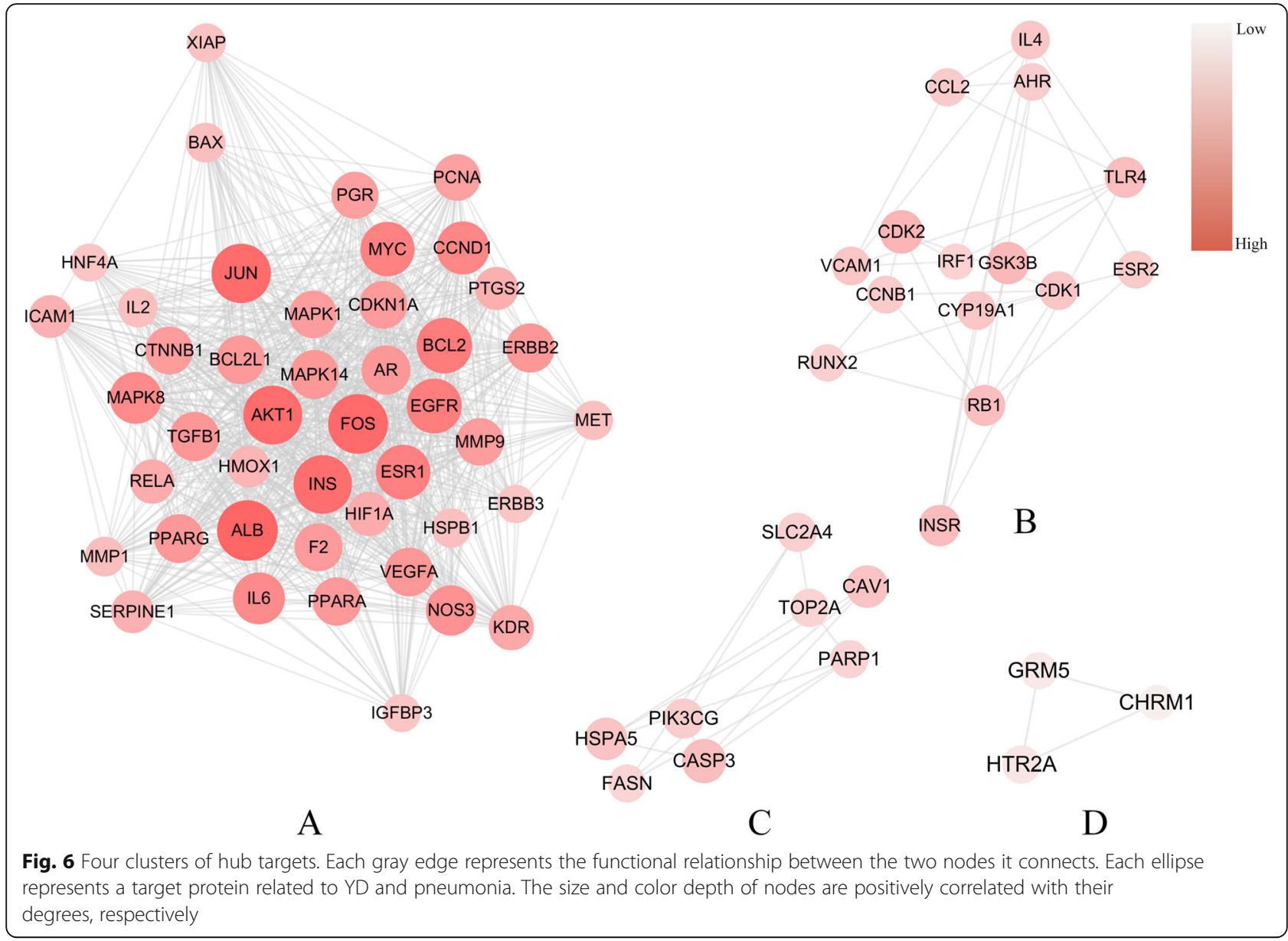

\section{Cell growth, proliferation, and apoptosis}

A variety of biological processes associated with cell growth, proliferation and apoptosis were returned, such as (GO:0042127) regulation of cell proliferation, (GO: 0042981) regulation of apoptosis, (GO:0010941) regulation of cell death, (GO:0043067) regulation of programmed cell death, (GO:0043066) negative regulation of apoptosis, (GO:0043069) negative regulation of programmed cell death, (GO:0060548) negative regulation of cell death, (GO:0006916) anti-apoptosis and so forth (Bonferroni< 0.05 ). Additionally, two high-degree pathways, (hsa04010) MAPK signaling pathway (degree = 11) and (hsa04110) Cell cycle (degree $=5$ ) were detected.

\section{Experiment validation}

\section{HE staining of lung tissue pathology}

According to the results of HE staining of lung tissue pathology, the structure of alveoli in NC group was intact and thin, without inflammatory infiltration, and the structure of bronchioles was intact and clear. Compared with $\mathrm{NC}$ group, the lung tissue structure in $\mathrm{P}$ group was damaged, and alveolar septum size and alveolar wall were thick and fragile. There was a large number of neutrophil infiltration and red cell extravasation in the lung interstation in P group. The structure of lung tissue and alveoli in PT group was clearer than that of P group, and the growth of pulmonary interstitial was reduced, with a small amount of inflammatory infiltration (as displayed in Fig. 9a).

\section{Cytokines of lung tissue}

The level of IL-6 of lung tissue was detected, which was significant higher in $\mathrm{P}$ group than $\mathrm{NC}$ group $(P<0.01)$. After YD treatment, the level of IL- 6 in the lung tissue decreased significantly $(P<0.01)$ (as displayed in Fig. $9 b)$.

\section{Discussion}

One of the common respiratory diseases is pneumonia. Due to drug resistance and undesired effects, limited options are currently available for its treatment. YD is a traditional Chinese medicine and extensively used in Chinese clinics for the treatment of pneumonia, mainly in children. However, its detailed mechanism of action is not discovered yet and thus attempted to explore in this network pharmacological study. 

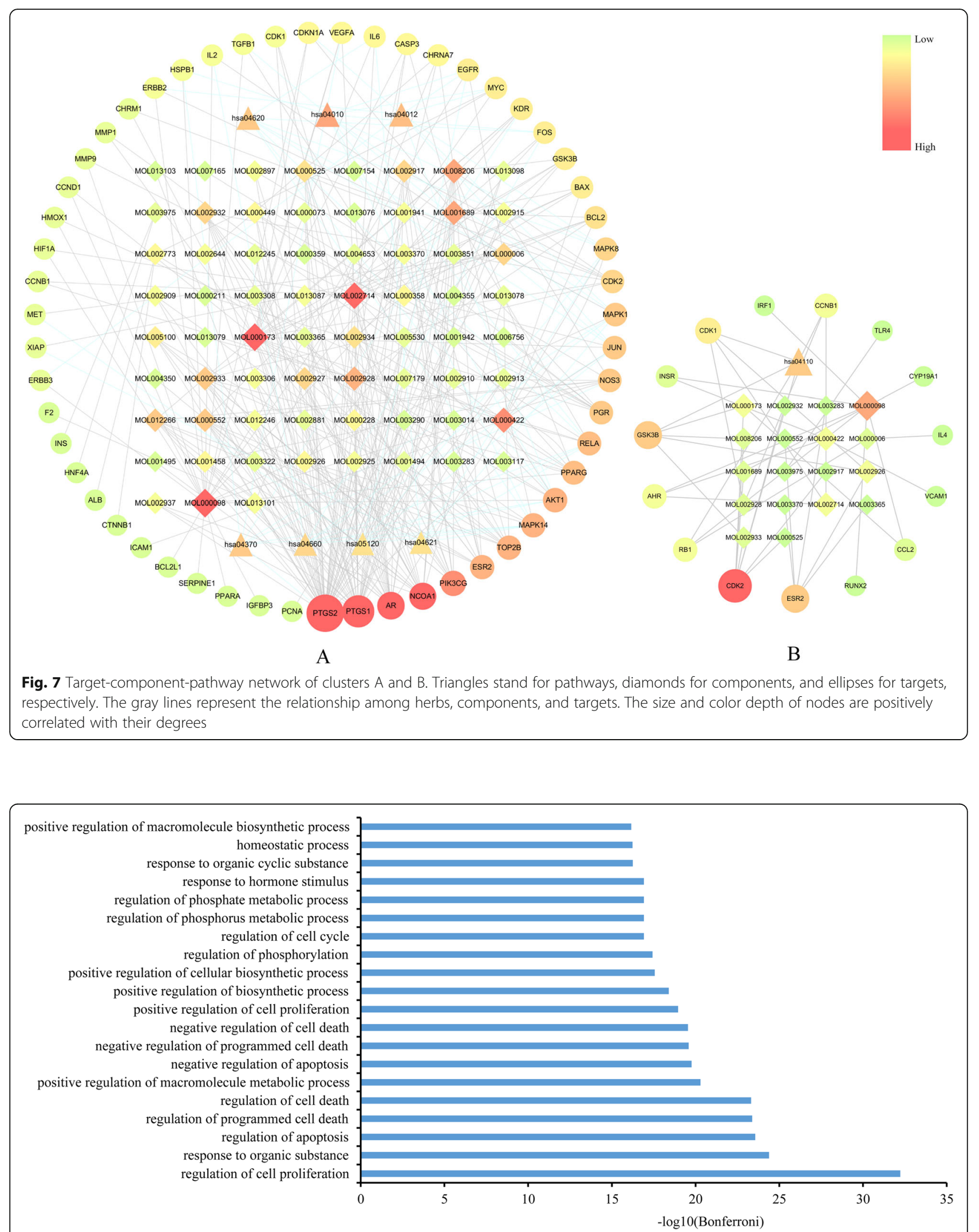

Fig. $8 \mathrm{GO}$ biological processes of YD for pneumonia. The top 20 biological processes are presented (Bonferroni< 0.05 ). It mainly involves the processes relevant to cell proliferation, apoptosis and death and metabolism of energy and substances 

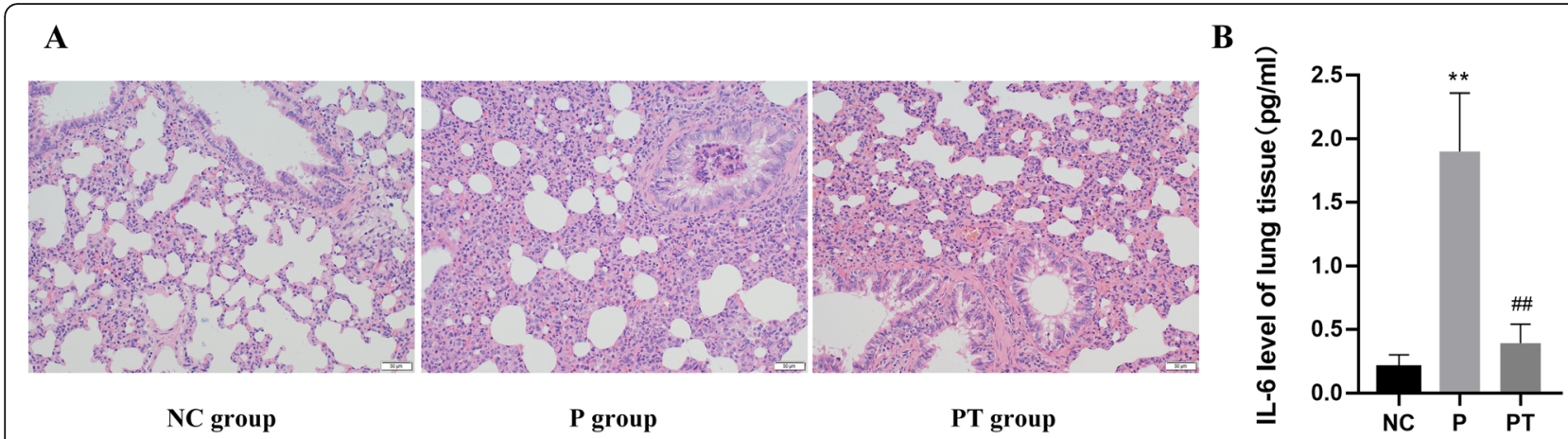

Fig. 9 Pathology and cytokines validation of lung tissue. a. Pathology of lung tissues by method of HE staining (200X). b. The cytokine level of IL6 in lung tissue. Values are represented as means \pm SEM, $n=6$ per group. ${ }^{*} P<0.05,{ }^{* *} P<0.01$ versus NC group, $\# P<0.05, \# \# P<0.01$ versus P group

\section{Anti-pathogenic and anti-inflammatory effects of YD ingredients}

This study revealed that YD had a large proportion of flavonoids such as luteolin, quercetin, wogonin, and kaempferol. Flavonoids possess a variety of pharmacological effects including antibacterial, anti-virus and antiinflammatory [30]. Researchers have found that desaminotyrosine (DAT) is a microbial metabolite of flavonoids, which can regulate type I interferon (IFN) signaling pathway and reduce lung pathological response for influenza treatment [31]. Immunomodulatory effects of wogonin on viral infection, lipopolysaccharides (LPS) stimulation and the imbalance of Th1/Th2/Th17 were observed [32]. Wogonin also directly attenuated the enzymatic activity of cyclooxygenase-2 (COX-2) at a low concentration [33]. The ability to inhibit nitric oxide synthase (NOS), COX-2 and $\mathrm{C}$ - reactive protein (CRP) protein was positively correlated with all concentrations of Kaempferol, but the inhibition effect of quercetin at high concentration was decreased [34]. This fact indicated that YD might exert anti-bacterial, anti-viral and anti-inflammatory activities in a certain dose-dependent manner.

\section{Two features of YD}

This study showed a multi-target feature of YD since 61 out of its 103 targets were related with several other targets (not less than 30), making a network and suggesting that the combined effects of multiple targets was responsible for the pharmacodynamics of YD. YD might modulate multiple systems to achieve the treatment of pneumonia. As shown in the network, hub targets of YD for treating pneumonia are not only concentrated on the modulation of the immune inflammatory response but also focused on the regulation of other accompanying factors related to cell proliferation, stress-activated cell survival, vascular system, and endocrine system [35-37].
The underlying mechanisms of YD in treating pneumonia TCM-based treatment system deals with the treatment of an individual (or a system) as a whole from an integrative and holistic way, in contrast to the "single-component and single-target" mode of the Western drugs. The drug resistance and side effects of Western drugs for pneumonia create an increasing trend of TCM therapy. Network pharmacology provides the researchers with a good paradigm. In this study, YD-pneumonia related targets and pathways were assembled to decipher the underlying mechanisms of YD in treating pneumonia from a systematic perspective. Since several targets can affect different pathways synchronously, resulting in a cross-regulation of multi-target and multi-pathway, current functional clusters were extracted and analyzed to assess the potential mechanisms associated with pneumonia (as displayed in Fig. 10).

\section{Relieving clinical symptoms of pneumonia}

The active components of YD have potential interaction with a variety of targets like PTGS1 and PTGS2, which are implicated in the mechanisms of multiple inflammatory diseases, including lung injury [38, 39]. The pulmonary inflammatory reaction is modulated by regulating prostaglandins (PGs) release that is controlled by respiratory epithelial cells. Cyclooxygenase (COX) is a key rate-limiting enzyme in PG biosynthesis. COX1 (PTGS1) is involved in the early stage of PGs physiological synthesis, whereas COX-2 (PTGS2), as the inducible isoform of COX, persists for a few hours after stimulation by an inflammatory response. Thus, YD might be involved in the alleviation of inflammatory reaction, fever, and pain by inhibiting PG synthesis.

\section{Regulation of hormone release}

It is noteworthy that several targets regulated by the herbs in YD like androgen receptor (AR) and ESR2, are implicated in the endocrine system. There is an 


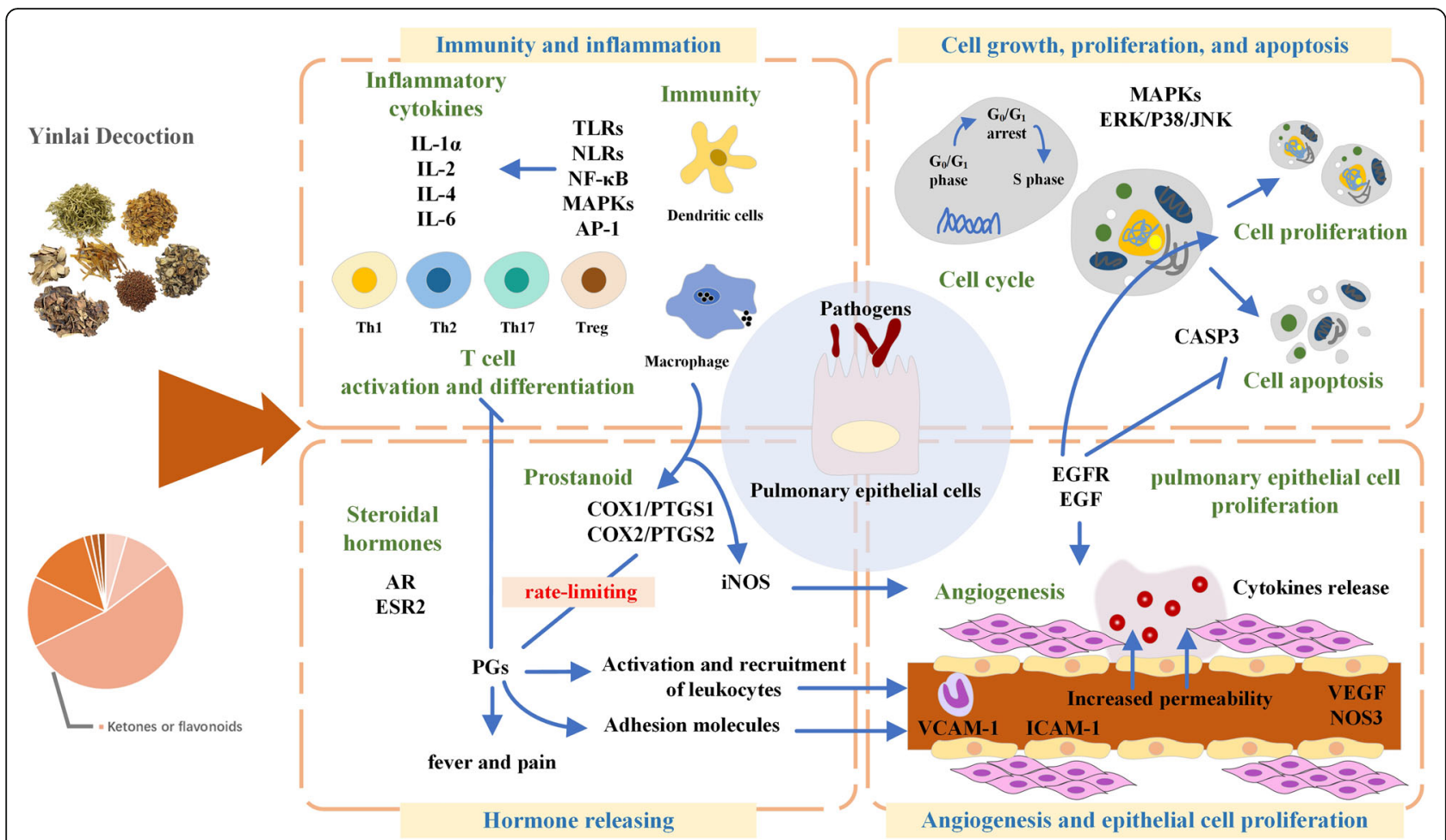

Fig. 10 Different mechanisms involved in the YD treatment of pneumonia. The triangular arrow represents activation, and the slanted arrow means inhibition. The activation and inhibition mechanisms showed in this figure are based on the knowledge from the literature and are not obtained from the results of any of the used platforms

increasing evidence that sex hormones possess pathophysiological actions in the lung. The gender-related difference in the morbidity and mortality of respiratory diseases has been gradually noted in clinical practice [40]. Previous studies have reported that exposure to cigarette smoke results in a higher likeliness of suffering from lung disorders with an up-regulated expression of AR contributing to a series of downstream pathological changes. It further explained the association between sex hormones and pulmonary diseases [41] and may act as another mechanism for the treatment of pneumonia by YD.

\section{Regulation of host immune inflammatory response}

It is a potential mechanism involved in the YD effect on the immune system. The recognition of pathogenassociated molecular patterns (PAMPs) by phagocytic cells and dendritic cells (DCs) is the first step to activate the host's immune system, ultimately contributing to the activation of intracellular signaling pathways and adaptive immune system. For the past few years, it has been convinced that the immune system primely perceives the environmental information after infection through Toll-like receptors (TLRs) expressed on the membrane of antigen-presenting cells (APCs) recognizing invasive pathogens. There are 10 different kinds of TLRs up to now [42, 43]. Because of TLRs' limited distribution,
NOD-like receptors (NLRs) can play an anti-infection role as intracellular receptors that respond to antigens. Therefore, NLRS can provide an identification system that may play a key role in the reaction of microbes or its cell wall components, which have already entered the cell or have escaped the surveillance of TLRs [44]. The APCs activated by TLR initiate innate and adaptive immune systems and promote the differentiation of T-cells to different directions of Th1, Th2, Th17 and Treg, which are closely related to the occurrence of various respiratory diseases such as pneumonia, influenza and asthma [45]. YD may treat pneumonia through regulating more than one pathway, which confirms that disorders are treated by using TCM in an integrative and holistic way.

\section{Intervention on angiogenesis and epithelial cell proliferation}

Several targets in EGFR, epidermal growth factor receptor (ErbB) and vascular endothelial growth factor (VEGF) signaling pathways involved in angiogenesis and epithelial cell proliferation are predicted in this study. The airway epithelial barrier defenses against the inhaled antigens and pathogens. EGFR plays a significant role in angiogenesis and the barrier function of the airway epithelium against pathogens, and simultaneously, lung 
epithelium TLRs expression initiate immune responses through triggering EGFR downstream signal cascade [46]. VEGF plays an important role in angiogenesis and maintenance of vascular permeability, which is one of the characteristic findings contributing to the respiratory tract remodeling. Staphylococcus aureus (S. aureus) and Streptococcus pneumoniae (S. pneumoniae) are two common factors for serious and complicated pneumonia. Researchers found that $S$. aureus and $S$. pneumoniae stimulated the release of VEGF from normal mesothelial cells and human neutrophils in a dose-dependent and time-dependent manner, respectively $[47,48]$. In addition, chronic inflammation and hyperimmune response are common characteristics of several lung diseases associated with Helicobacter pylori (Hp) infection, including chronic bronchitis, asthma, and lung cancer. Researchers found that Hp-infected patients might suffer from an increased risk of pneumonia for high gastric $\mathrm{pH}$ levels caused by Hp infection and gastric acid inhibitor [49]. Above all, the results indicate that YD is effective in the treatment of pneumonia through regulating angiogenesis and epithelial cell proliferation.

\section{Regulation of cell cycle arrest and apoptosis}

Several potential targets, such as JNK1 (MAPK8), ERK2 (MAPK1) and p38- $\alpha$ (MAPK14) participate in mitogenactivated protein kinase (MAPK) pathways which act as a regulator of cell cycle arrest and apoptosis [50] and play an important role in signal transduction during the inflammatory reaction [51]. As we all know, the replication of the virus occurs by regulating the cell cycle progression of the host, which is a feature of their pathogenic mechanism. As a common pathogeny of pediatric pneumonia and bronchitis, an efficient replication of the respiratory syncytial virus (RSV) can be ensured by mediating cell cycle arrest via increasing transforming growth factor- $\beta$ (TGF- $\beta$ ) and MAPK signaling pathway [52]. Thus, the results display another potential mechanism that YD may target MAPK signaling pathways and cell cycle to inhibit respiratory viral replication and signal transduction during the inflammatory response.

The systematic role of YD in the treatment of pneumonia In summary, the results of network construction and analysis demonstrate that YD, as a multi-target agent, comprises multiple components and alleviates symptoms of pneumonia through regulating host immune inflammatory response (like TLRs, nuclear factor-kappa B (NF$\kappa B)$ and IL-6), angiogenesis and vascular permeability [such as VEGF, NOS3, vascular cell adhesion molecule-1 (VCAM -1) and intercellular adhesion molecule 1 (ICAM-1)], barrier function of the airway epithelial cells (like EGFR and EGF), hormone-releasing (such as AR,
ESR2, PTGS1, and PTGS2), and the pivotal proteins in the process of cell cycle and signal transduction (such as JNK, ERK and p38). These biological processes and pathways are not isolated, but connected, interacting with each other to target pneumonia.

\section{Limitation of network pharmacology}

There are still a few unknown compounds and their targets, thus the pharmacological effects of an herbal formula cannot be revealed completely. Another limitation is the false negative due to the targets rooted from a different database, which may have biased impact because of different experimental conditions. We are also aware of the public databases that have limited information. At present, some public databases tend to focus on some hot researches, so complete pharmacological effects of some other disease cannot be revealed.

\section{Experimental verification}

Network pharmacology showed that IL-6 was a remarkable predicted target which was related with infection. In the inflammatory reaction, the rise of IL-6 was earlier than other cytokines, and lasted for a long time, so it can be used to assist the early diagnosis of acute infection. After bacterial infection, the level of IL- 6 increased rapidly and reached the peak at $2 \mathrm{~h}$, which was consistent with the severity of infection. Therefore, IL-6 has more advantages as an inflammatory marker. It showed that YD could significantly alleviate the inflammatory changes in the lung and reduce the expression of IL-6 in the lung tissue.

\section{Conclusion}

Upon the above findings, we assume that YD may not directly target pathogens but exerts its therapeutic effect against pneumonia by adjusting the pulmonary environment and overall state of the host. YD turns out to be a potential "multi-component and multi-target" agent, providing a novel approach for clinical practice. However, our work only dissects some potential therapeutic mechanisms of YD and a small amount of verification work has been carried out. Further experimental verification is still necessary for the future.

\section{Supplementary information}

Supplementary information accompanies this paper at https://doi.org/10. 1186/s12906-020-02954-z.

Additional file 1: Table S1. Active ingredients and ADME parameters of YD. Table S2. Target information of related active ingredients. Table S3. The relationship between active ingredients and potential targets.

\section{Abbreviations}

DL: Druglikeness; GL: Trichosanthes rosthornii Harms; IL: Interleukin; HE: Hematoxylin-eosin; HQ: Scutellaria baicalensis Georgi; JYH: Lonicera 
japonica Thunb; LFZ: Raphanus sativus L; LQ: Forsythia suspensa (Thunb.) Vahl; NC: Normal contral; OB: Oral Bioavailability; P: Pneumonia; PPI: Proteinprotein interaction; PT: Pneumonia tratment; $\mathrm{QH}$ : Peucedanum praeruptorum Dunn; TCM: Traditional Chinese medicine; YD: Yinlai Decoction;

YXC: Houttuynia cordata Thunb

\section{Acknowledgements}

Not applicable.

\section{Authors' contributions}

J.X., C.B. and L.H. conceived and designed the study. J.X., C.B., L.H. and X.M. carried out the data preparation and topological analysis. J.X., C.B., L.H. and F.G. undertook network construction and analysis. Y.W. and Z.Z. provided technical assistance. J.X., T.L. and X.G. helped with manuscript drafting and revised the manuscript. X.G. and T.L. provided the financial assistance. H.Y. helped with the language. All authors reviewed the manuscript. The author(s) read and approved the final manuscript.

\section{Funding}

The research is financially supported by the National Natural Science Foundation of China (Grant no. 81874421) and Beijing Nova Program (Grant no. xx2018056, Z181100006218083). They both participated in the collection, analysis, and interpretation of data.

\section{Availability of data and materials}

The datasets generated and/or analyzed during the current study are available from the corresponding author on reasonable request.

\section{Ethics approval and consent to participate}

Not applicable.

\section{Consent for publication}

Not applicable.

\section{Competing interests}

The authors declare no competing interests.

\section{Author details}

${ }^{1}$ School of Traditional Chinese Medicine, Beijing University of Chinese Medicine, Beijing 100029, People's Republic of China. ${ }^{2}$ Department of Acupuncture and Mini-invasive Oncology, Beijing University of Chinese Medicine Third Affiliated Hospital, Beijing 100029, People's Republic of China. ${ }^{3}$ School of Basic Medical Sciences, Guiyang University of Chinese Medicine, Guiyang, Guizhou 550025, People's Republic of China.

Received: 18 July 2019 Accepted: 14 May 2020

Published online: 03 June 2020

\section{References}

1. Lanks CW, Musani Al, Hsia DW. Community-acquired pneumonia and hospital-acquired pneumonia. Med Clin North Am. 2019;103(3):487-501.

2. Zhang $\mathrm{S}$, et al. Cost of management of severe pneumonia in young children: systematic analysis. J Glob Health. 2016;6:010408.

3. Jin Y, Yang H, Ji W, et al. Virology, epidemiology, pathogenesis, and control of COVID-19. Viruses. 2020;12(4):E372.

4. Taussig LM, M. Pediatric respiratory medicine. Second ed; 2008

5. Liu TG, et al. Influences of Yinlai Tang on slgA, TNF-a and IL-10 in intestinal mucosal tissues of mice with dyspepsia combined with influenza virus infection. J Beijing Univ Tradit Chinese Med. 2014;37:86-9.

6. Gao Y, et al. A network pharmacology approach to decipher the mechanisms of anti-depression of Xiaoyaosan formula. World J Tradit Chinese Med. 2018;4:147-62.

7. Gao K, et al. Effects of Qijian mixture on type 2 diabetes assessed by Metabonomics, gut microbiota and network pharmacology. Pharmacol Res. 2018;130:93-109.

8. Xiao S, et al. A visual analysis of network pharmacology research trends. World J Tradit Chinese Med. 2018:4:163-9.

9. Zhang SD, et al. Exploring the pathways and targets of Shexiang Baoxin pill for coronary heart disease through a network pharmacology approach. World J Tradit Chinese Med. 2018;4:137-46.
10. $R u$ JL, et al. TCMSP: a database of systems pharmacology for drug discovery from herbal medicines. J Cheminform. 2014;6:13.

11. Xu X, Zhang W, Huang C, Li Y, Yu H, Wang Y, Duan J, Ling Y. A Novel Chemometric Method for the Prediction of Human Oral Bioavailability. Int J Mol Sci. 2012;13:6964-82.

12. Ano R, Kimura YM, Matsuno R, Ueno T, Akamatsu M. Relationships between structure and high-throughput screening permeability of peptide derivatives and related components with artificial membranes: application to prediction of caco-2 cell permeability. Bioorg Med Chem. 2004;12:257-64.

13. Ritchie TJ, Macdonald SJ. How drug-like are 'ugly' drugs: do drug-likeness metrics predict ADME behaviour in humans? Drug Discov Today. 2014;19(4): 489-95.

14. Gong J, et al. ChemMapper: a versatile web server for exploring pharmacology and chemical structure association based on molecular 3D similarity method. Bioinformatics. 2013;29:1827.

15. Consortium UP. UniProt: a hub for protein information. Nucleic Acids Res. 2015;43:204-12.

16. Li $\mathrm{YH}$, et al. Therapeutic target database update 2018: enriched resource for facilitating bench-to-clinic research of targeted therapeutics. Nucleic Acids Res. 2017:46:D1121-D1127.

17. Law V, et al. DrugBank 4.0: shedding new light on drug metabolism. Nucleic Acids Res. 2014;42:1091-7.

18. Köhler $\mathrm{S}$, et al. The human phenotype ontology in 2017. Nucleic Acids Res. 2017;45:D865-76.

19. Hamosh A, et al. Online Mendelian inheritance in man (OMIM), a knowledgebase of human genes and genetic disorders. Nucleic Acids Res. 2005:33:514-7.

20. Grondin CJ, Davis AP, Wiegers TC, Wiegers JA, Mattingly CJ. Accessing an expanded exposure science module at the comparative Toxicogenomics database. Environ Health Perspect. 2018;126:014501.

21. Szklarczyk D, et al. STRING v10: protein-protein interaction networks, integrated over the tree of life. Nucleic Acids Res. 2015;43:D447.

22. Lopes $C T$, et al. Cytoscape web: an interactive web-based network browser. Bioinformatics. 2010;26:2347-8.

23. Huang DW, Sherman BT, Lempicki RA. Systematic and integrative analysis of large gene lists using DAVID bioinformatics resources. Nat Protoc. 2008:4:44

24. Fatma M, Mohamed S. Enhanced anticancer activity and oral bioavailability of ellagic acid through encapsulation in biodegradable polymeric nanoparticles. Int J Nanomedicine. 2017;12:7405-17.

25. Lu T, et al. Plasma and urinary tanshinol from Salvia miltiorrhiza (Danshen) can be used as pharmacokinetic markers for cardiotonic pills, a cardiovascular herbal medicine. Drug Metabol Dispos Biol Fate Chem. 2008; 36:1578.

26. Liu WC, et al. A network perspective on the topological importance of enzymes and their phylogenetic conservation. Bmc Bioinformatics. 2007;8(1): 121.

27. Jordán F, Liu WC, Memmott DJ. Topological keystone species: measures of positional importance in food webs. Oikos. 2006;112(3):535-46.

28. Holme $\mathrm{P}$, Huss $\mathrm{M}$, Jeong $\mathrm{H}$. Subnetwork hierarchies of biochemical pathways. Bioinformatics. 2003;19(4):532-8.

29. Ma HW, Zeng AP. The connectivity structure, gian strong component and centrality of metabolic networks. Bioinformatics. 2003;19(11):1423-30.

30. Shohaib T, Shafique M, Dhanya N, Divakar MC. Importance of Flavonoides In therapeutics. Hygeia J Drugs Med. 2011;3(1):1-18.

31. Steed $A L$, et al. The microbial metabolite desaminotyrosine protects from influenza through type I interferon. Science. 2017;357:498.

32. Takagi $R$, et al. Wogonin attenuates ovalbumin antigen-induced Neutrophilic airway inflammation by inhibiting Th17 differentiation. Int J Inflamm. 2014;2014:571508.

33. Wakabayashi I, Yasui K. Wogonin inhibits inducible prostaglandin E 2 production in macrophages. Eur J Pharmacol. 2000;406:477-81.

34. García-Mediavilla $\vee$, et al. The anti-inflammatory flavones quercetin and kaempferol cause inhibition of inducible nitric oxide synthase, cyclooxygenase2 and reactive C-protein, and down-regulation of the nuclear factor kappaB pathway in Chang liver cells. Eur J Pharmacol. 2007:557:221-9.

35. Zhong X, Kulis M, Guo R, Pons L, Burks AW. Streptococcus pneumoniae induced C-Jun-N-terminal kinase- and AP-1 -dependent IL-8 release by lung epithelial BEAS-2B cells. Respir Res. 2006;7:98.

36. Li XM, et al. Study on JNK/AP-1 signaling pathway of airway mucus hypersecretion of severe pneumonia under RSV infection. Eur Rev Med Pharmacol Sci. 2016;20:853. 
37. Yang $Z$, et al. Female resistance to pneumonia identifies lung macrophage nitric oxide synthase-3 as a therapeutic target. Elife. 2014;3.

38. Fukunaga K, Kohli P, Bonnans C, Fredenburgh LE, Levy BD. Cyclooxygenase 2 plays a pivotal role in the resolution of acute lung injury. J Immunol. 2005; 174:5033-9.

39. Zhang $L N$, et al. Protective effects of asiaticoside on septic lung injury in mice. Exp Toxicol Pathol Official J Gesellschaft Für Toxikologische Pathologie. 2011;63:519.

40. Chang C, Lee SO, Yeh S, Chang TM. Androgen receptor (AR) differential roles in hormone-related tumors including prostate, bladder, kidney, lung, breast and liver. Oncogene. 2014;33:3225-34.

41. Sun $\mathrm{CH}$, et al. Effect of Acrolein on the protein expression of androgen receptor in male rat lung. Biol Reprod. 2012;87:251.

42. Branger J, et al. Role of toll-like receptor 4 in gram-positive and gramnegative pneumonia in mice. Infect Immun. 2004:72:788.

43. Wright SD, Ramos RA, Tobias PS, Ulevitch RJ, Mathison JC. CD14, a receptor for complexes of lipopolysaccharide (LPS) and LPS binding protein. Science. 1990;249:1431-3.

44. Abrahams V, Abrahams M. V.M. the role of the nod-like receptor family in trophoblast innate immune responses. J. Reprod. Immunol. 88, 112-117. J Reprod Immunol. 2011;88:112-7.

45. Mangodt TC, et al. The role of Th17 and Treg responses in the pathogenesis of RSV infection. Pediatr Res. 2015;78:483-91.

46. Koff JL, Shao MX, Ueki IF, Nadel JA. Multiple TLRs activate EGFR via a signaling cascade to produce innate immune responses in airway epithelium. Am J Physiol Lung Cell Mol Physiol. 2008;294:L1068.

47. Mohammed KA, et al. Bacterial induction of pleural mesothelial monolayer barrier dysfunction. Am J Physiol Lung Cell Mol Physiol. 2001;281:119-25.

48. Van DFM, Coenjaerts F, Kimpen JL, Hoepelman AM, Geelen SP. Streptococcus pneumoniae induces secretion of vascular endothelial growth factor by human neutrophils. Infect Immun. 2000;68:4792-4.

49. Kanbay M, Kanbay A, Boyacioglu S. Helicobacter pylori infection as a possible risk factor for respiratory system disease: a review of the literature. Respir Med. 2007;101:203-9.

50. Wang $\mathrm{D}$, et al. A new prenylated flavonoid i nduces $\mathrm{g} 0 / \mathrm{g} 1$ arrest and apoptosis through p38/jnk mapk pathways in human hepatocellular carcinoma cells. Sci Rep. 2017;7(1):5736.

51. Karin W, Nam V, Dimitris K, Norbert B. Inflammatory and catabolic signalling in intervertebral discs: the roles of NF-KB and MAP kinases. Eur Cells Mater. 2012;23:103.

52. Gibbs JD, et al. Cell cycle arrest by transforming growth factor $\beta 1$ enhances replication of respiratory syncytial virus in lung epithelial cells. J Virol. 2009; 83:12424-31.

\section{Publisher's Note}

Springer Nature remains neutral with regard to jurisdictional claims in published maps and institutional affiliations.

Ready to submit your research? Choose BMC and benefit from:

- fast, convenient online submission

- thorough peer review by experienced researchers in your field

- rapid publication on acceptance

- support for research data, including large and complex data types

- gold Open Access which fosters wider collaboration and increased citations

- maximum visibility for your research: over $100 \mathrm{M}$ website views per year

At BMC, research is always in progress.

Learn more biomedcentral.com/submissions 